infection in a number of children selected for operation. As Mr. Walker suggests in his final comments, this resolves in the vast majority of cases after removal of the diseased tonsils and adenoids. He considers that the unsatisfactory results following tonsillectomy and adenoidectomy are due to sinusitis : we consider that they are more likely to be due to an incorrect selection of children for operation.-We are, etc.,

\section{R. SHeridan.}

Truro, Cornwall.

\section{T. M. BANHAM.}

\section{Residual Prostatitis}

SIR,-We have read with great interest Dr. F. C. Bourgault Du Coudray's excellent article on residual prostatitis (Oct. 25, 1947, p. 651). Such a condition has been observed by us in a large number of cases, and assuredly also by many others, after sulphonamide or penicillin treatment of the urethral-process gonorrhoea. Some cases were treated for months and even years with massage, diathermy, and high supplementary doses of antibiotics notwithstanding the fact that repeated cultures of prostatic fluid and of semen were sterile or showed some diphtheroids or Gram-positive cocci or bacilli. Streptomycin was used without success in 2 cases.

The persistence of pus cells in the prostatic fluid indicated the existence of an infection whose true nature we have cleared up in quite a few cases after dark-field observation of the prostatic fluid. In a recent communication to the III PanAmerican Congress of Urology (Rio de Janeiro, Sept. 14-20, 1947) we referred to 50 cases of such a type of prostatitis observed in V.D. clinic patients ; in $6(12 \%)$ we found spirilla, some morphologically similar to $S p$. dentium. Latterly we have found in 7 other cases spirilla, in $3 \mathrm{Tr}$. vaginalis, and in 2 an, association of both these micro-organisms. All cases were cured rapidly with arsenical therapy-trivalent, pentavalent, or arsenoxide.

In 3 out of 10 cases in which we did not find spirochaetes or protozoa we obtained rapid and remarkable results with the arsenical therapy, based on the knowledge that the former microorganisms may disappear, breaking up into coccoid or granular forms (Dutton, Leishman, Balfour, Nicolle, Coutts, Marchoux, Chorine, etc.).

As regards the negative action of penicillin in cases where spirochaetes have been found, it is of convenience to remark that this drug does not act equally on the different types and that even $S p$. pallida sometimes requires very large doses in order to be destroyed.

When prostatic fluid contains numerous pus cells we dilute it with tepid normal saline and examine a drop of the dilution. An excess of pus cells impedes the free movements of spirochaetes and of the cilia of trichomonads, rendering their search almost impossible. Following this practice we have frequently been able to demonstrate the presence of these microorganisms in fluids that had been declared sterile.-We are, etc.,

Santiago de Chile

$$
\text { Waldemar E..COUTtS. }
$$$$
\text { EDNA Silva-INZUNZA. }
$$

\section{Cheaper Heparin}

SIR,--Recent correspondence on heparin and dicoumarol prompts me to write on the subject of these anticoagulant drugs. Whatever may be the views held by physicians on the value of anticoagulants in the treatment of coronary thrombosis, surgeons should be in no doubt about the value of anticoagulant therapy, properly managed, in cases of postoperative venous thrombosis and embolism. In vlew of this fact, it is perhaps surprising that these drugs are not more widely used.

There would seem to be two reasons for the present state of affairs: on the one hand we have heparin, a highly effective, safe, and expensive drug, while on the other hand there is dicoumarol, a cheap, potentially dangerous, but more readily obtainable drug. Experience with dicoumarol seems to vary greatly, and, although one knows some who have used it without experiencing any trouble, there are many who report serious and even fatal complications following its use. While it is clear that the most careful laboratory control is essential in dicoumarol therapy, it is doubtful whether a substance which has been described by no less an authority than Jorpes as exerting "a kind of toxic narcosis of the liver cells, the depth of which it is difficult to control," is really the drug of choice in the treatment of thrombosis.
At the present time an average day's treatment with heparin costs more than five pounds, whereas that with dicoumarol may cost less than a shilling. Under these circumstances it is hardly surprising that dicoumarol is sometimes tried (often, it is true, under inadequate laboratory control), found wanting. and discarded, while heparin, being expensive and in short supply, is used far too little.

Since the great value of heparin should need no emphasis I would like, through your columns, to put in a strong plea for the manufacture of a plentiful, cheap, and reliable brand of heparin. Doubtless other readers will be ready to express their views and, if necessary, to encourage the commercial drug firms to satisfy our needs. - I am, etc.,

Radlett, Herts.

Reginald S. MurLey.

\section{Drug Addiction}

SIR, - l was interested in your annotation on drug addiction (Dec. 13, 1947, p. 965). The group of opium eaters who appear to be in an "arrested development " stage of addiction must be familiar to medical officers who served with Indian troops in the recent war. It was found that Indian troops, who were able to obtain supplies of opium in India quite easily, presented a problem when posted out of India to countries where opium was not so readily obtainable. At first opium eaters were posted back to India, but later policy was changed, and these men were supplied with a limited amount of crude opium up to a maximum of $300 \mathrm{gr}$. $(20 \mathrm{~g}$.) per month.

In my unit half a dozen men were kept efficient and healthy with such a maintenance dose. Some received 200 gr. $(13$ g. $)$. the others the full 300 gr. monthly. I followed these men over a period of $2 \frac{1}{2}$ years and found that their work was satisfactory in every way ; they did not make frequent appearances at sick parade nor did they seek increasing doses of opium to remain in such equilibrium. I made attempts to break them of the habit, but since they were men over 30 with a history of ten years or more of taking the drug success was neither anticipated nor achieved.

On the other hand, a dozen or more young soldiers posted to the unit direct from India were broken of the habit quite easily by immediate withdrawal of the drug. This group suffered virtually no withdrawal symptoms and emphasizes the point brought out by your annotation-that it is a mistake to apply the term "drug addiction" to all cases in which drugs are taken merely to produce euphoria.- I am etc.

$$
\text { Bristol. }
$$

$$
\text { Philip Jacobs. }
$$

\section{Child-bearing and Tuberculosis}

Sir,-Dr. C. J. Stewart and Dr. F. A. H. Simmonds (Nov. 8. 1947 , p. 726) have noted that the majority of physicians who have recent experience in the matter agree that pregnancy does not activate tuberculous disease : they have confirmed this themselves by a statistical analysis of a large series of patients. and they have drawn the conclusion that abortion is not a therapeutic agent in tuberculosis. Their comment in your issue of Dec. 27, 1947 (p. 1056), that effective advice concerning birth control had not been given to the patients in their series. and their suggestion that such advice is advisable especially for two years after the disease has become quiescent, is less logical, because they have already shown that in spite of lack of such advice, and also the lack of therapeutic abortion, the patients with active disease in their series who were pregnant compared not unfavourably in results with a control group with active disease who were not pregnant. Dr. R. C. Cohen found in his analysis of the Black Notley cases that a woman who became pregnant soon after quiescence of the disease would as likely do well as a woman who became pregnant later.

Admittedly, unlimited pregnancies should not be encouraged in tuberculous women. But the psychosomatic background of tuberculous disease is becoming increasingly perceptible. Conjugal felicity is important for a tuberculous woman, as for others, and if by effective birth control is meant artificial contraception it is interesting to speculate whether our generation, to whom advice in artificial contraception has not been wanting. is the more blessed in happy marriages. Might it not rather be better for the tuberculous woman and her husband to avoid a facile solution and by mutual co-operation and sacrifice to grow in that mutual respect on which happy marriages are built? 